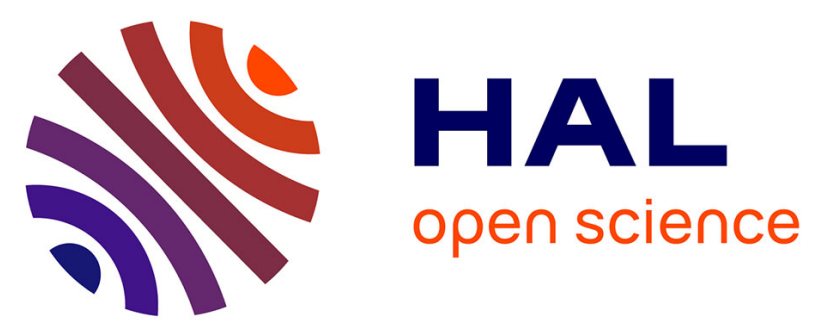

\title{
Exercices de style pour hypothèses courtoises: les chansons 1 et 16 et la lettre V de Guittone d'Arezzo (vers 1260-1265?)
}

Anne Robin

\section{- To cite this version:}

Anne Robin. Exercices de style pour hypothèses courtoises: les chansons 1 et 16 et la lettre $\mathrm{V}$ de Guittone d'Arezzo (vers 1260-1265?). Arzanà. Cahiers de littérature médiévale italienne, 2007, Poésie et épistolographie dans l'Italie médiévale. Etudes réunies par A. Fontes-Baratto., 12, p. 37-63 (dont annexe: textes traduits). hal-01395722

HAL Id: hal-01395722

https://hal.univ-lille.fr/hal-01395722

Submitted on 11 Nov 2016

HAL is a multi-disciplinary open access archive for the deposit and dissemination of scientific research documents, whether they are published or not. The documents may come from teaching and research institutions in France or abroad, or from public or private research centers.
L'archive ouverte pluridisciplinaire HAL, est destinée au dépôt et à la diffusion de documents scientifiques de niveau recherche, publiés ou non, émanant des établissements d'enseignement et de recherche français ou étrangers, des laboratoires publics ou privés. 


\section{ARZANÀ 12}

\section{Presses Sorbonne Nouvelle}

8 rue de la Sorbonne - 75005 Paris

Tel : 0033 (0)140464802 - Fax : 0033 (0)1 40464804

Courriel : psn@univ-paris3.fr

http://psn.univ-paris3.fr

UNIVERSTTE. SORBONNE NOUVELLE - PARIS 3

UFR D'ÉTUDFS TTALIENNES ET ROUMAINES

Centre d'ÉTUdes ta de ReCHERCHES SUR

la littératuke. Itatifenne Médiévale (CERLIM) dans l'Ttalie médiévale

Études réunies et présentées par Anna FonTES BARATTO

Mise en page : Laurent Tournier

(C) PRESSISS SORBONNE NOUVELLE

ISSN 1243-3616

Sorbonnnes

ISBN $978-287854-382-7$ 


\section{Table des matières}

Anna Fontes Baratto

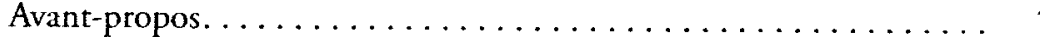

Cécile le Lay,

Une lettre pour « se rétracter " après la Chanson de Montaperti? . . . 17

Anne Robin,

Exercices de style pour hypothèses courtoises:

les chansons 1 et 16 et la lettre V de Guittone d'Arezzo

(vers 1260-1265?)

Anna Fontes Baratto

Le diptyque montanino de Dante.

SABRINA FERRARA,

La métaphore solaire.

Les trois épîtres politiques de Dante et le chant XVI du Purgatoire . .

Véronique AbBruZzetTi,

Pétrarque et son fantôme.

\section{Marina Gagliano,}

Prophétisme et polémique dans les sonnets babyloniens

et dans les épîtres Sine nomine de Pétrarque.

ISABELle BATTESTI,

Parler de soi à soi seul :

l'épitre métrique I, 14 Ad se ipsum et la canzone 264 de Pétrarque. . . 153
Patrizia Gasparini,

Non mi posso tener più cbio non dica:

Franco Sacchetti entre invective politique et blâme moral

(Lettre XI, Chanson 141)

TABLE DES ANNEXES : TEXTES ET TRADUCTIONS 


\section{Exercices de style pour hypothèses courtoises : les chansons 1 et 16 et la lettre $V$ de Guittone d'Arezzo (vers 1260-1265?)}

Wous donnerons d'emblée le ton de cette étude des chansons 1 et 16 et de la lettre $V$ de Guittone d'Arezzo en citant une phrase d'E. Pasquini : la "prose et la poésie guittoniennes se rejoignent à un niveau d'audace exceptionnelle sans pareille à l'époque ". Le critique mentionne d'ailleurs la chanson 1 et la lettre $\mathrm{V}$ comme appartenant au " territoire commun " des Rimes et des Lettres. Nous leur avons adjoint ici, pour les raisons que nous verrons, la chanson 16.

Quel est le "territoire commun" auquel fait allusion Pasquini? Il ne s'agit ni du "mètre ", ni du genre littéraire. Les deux chansons en hendécasyllabes et settenari rimés, organisées selon un schéma classique - cinq strophes de vingt et un vers pour la première, et de douze pour la seizième, suivies d'un congé plus ou moins long dont le schéma métrique est régulièrement identique à la sirma des strophes - appartiennent à la poésie lyrique. La lettre, tout en étant "riche en clausules et en isocolies", mais non rimée, contrairement à d'autres qui vont jusqu'à adopter le schéma de la chanson (les lettres VI, XI, XXX par exemple), suit les règles des manuels d'épistolographie. Elle s'ouvre sur une salutatio (le paragraphe 1), se poursuit sur la propositio $(\$ 2)$; et viennent ensuite la narratio $(\$ 3)$, la conclusio

1. « $\grave{E}$ ben noto che nella tradizione mediolatina "i limiti fra poesia e prosa vennero progressivamente cancellati"; ma è altrettanto vero che prosa e poesia guittoniane s'incontrano a un livello di innaturale spericolatezza che non trova riscontri ai suoi tempi. " Emilio Pasquini, "Intersezioni fra prosa e poesia nelle Lettere di Guittone d'Arezzo ", in Guittone d'Arezzo nel settimo centenario della morte, Att del Convegno Internazionale di Arezzo (22-24 aprile 1994), a cura di Michelangelo Picone, Firenze, Franco Cesati Editore, 1995, p. 183. 
$(\$ 4)$ et la petitio $(\$ 5-7)^{2}$. Ce territoire commun n'est pas non plus d'ordre thématique, au contraire des célèbres chanson 19 et lettre XIV qui traitent toutes deux de la défaite de Montaperti. Alors quel est-il ? À première vue il comprend la situation initiale du discours évoquée à partir de syntagmes plus ou moins similaires et que nous pouvons décrire ainsi : le " je » loue la dimension, sinon miraculeuse, du moins merveilleuse, de la dame à qui il s'adresse. Dans la suite cependant, toujours à première vue, les chansons et la lettre divergent voire s'opposent, telles la chanson 16 et la lettre. Une étude approfondie des textes s'impose donc.

Celle-ci va mettre en évidence une communauté plus grande que celle se manifestant dès l'abord. Nous verrons en effet que l'unité s'étend au type de dame à qui s'adresse le discours et à l'identité du locuteur, et qu'elle concerne aussi la fonction des textes.

$$
\infty
$$

Bien que les deux chansons appartiennent à l'œuvre courtoise de Guittone, tandis que la lettre appartient à son œuvre religieuse et morale, le discours des trois textes s'adresse au même type de dame : une "mirabel cosa " que le cœur humain ne peut imaginer (c. 1, v. 21 et 13; c. 16, v. 3-4); un être surnaturel qui demeure ici-bas et dont ce qu'on voit "paraît miraculeux à ceux qui sont bons juges" (c. 1, v. 19 et $21 ; 1 . \mathrm{V}, \S 2)$; un être à l'essence et au comportement "par-dessus tout plaisant " (c. 16, v. 2; I. V, $\$ 1$ ) et « honorable " (c. $16, v .2 ; 1 . \mathrm{V}, \$ 5$ et 6 ); une dame " en tout point accomplie" (c. 16, v. $3 ; 1 . V \$ 1$ et 2$)^{3}$.

2. Citation et division de Claude Margueron, in Guntrone d'Arezzo, Lettere, a cura Cl. Margueron, Bologna, Commissione per i testi di lingua, 1990, p. 87-88. La lettre VI est une chanson d'une seule très longue strophe de 32 vers mêlant hendécasyllabes et settenari. La lettre XI est une chanson lacunaire de trois strophes de 17 vers suivies d'un congé reprenant le schéma métrique de la fin de la sirma des strophes. La lettre XXX est une chanson de trois strophes de 19 vers.

3. Voici les trois contextes. Nous avons utilisé des styles différents pour souligner les points communs. Les textes des chansons citent l'édition Le rime di Guittone d'Arezzo, a cura di Franco Egidi, Bari, Laterza, 1940. Le texte de la lettre est cité d'après l'édition de Cl. Margueron :

Ahi, Deo, co sì novella

pote a esto mondo dimorar figura,
L'adjectif "compiuta» qualifiant la dame de la lettre a été généralement interprété, non pas comme l'expression littérale d'une qualité, mais comme le senhal de la poétesse connue sous le nom de Compiuta Donzella di Firenze. Cette identification proposée entre autres par G. Contini et M. Marti est tenace ${ }^{4}$, mais il n'y a aucun indice permettant d'érabli un lien entre la destinataire de la lettre et la poétesse. Pour Cl. Margueron cette dame est fictive ${ }^{5}$. Les points communs que nous avons mis en évidence entre nos trois textes confirment assurément cette hypothèse : la destinataire de la lettre $V$ n'est pas la Compiuta Donzella di Firenze mais le même type de dame que celles auxquelles s'adresse le discours courtois des chansons 1 et 16

Intéressons-nous maintenant au locuteur des chansons et de la lettre Le " je " anonyme des deux chansons est l'amant traditionnel de la poésic courtoise, un amant qui, traditionnellement aussi, met en avant sa fidélité (c. 1, v. $47-48 ;$ c. 16, v. 68). Le « je " de la lettre se présente comme « Guitton", mais qui est ce "Guittone"? Est-ce, comme l'indique la rubrique

\section{ch'è de sovra natura?}

Ché_ciò che l'om de yoi conosce e vede.

semiglia, per mia fede,

mirabel sosa_a_bon conoscidore. (c. 1, v. 16-21)

Gentil mia donna, gioi sempre gioiosa,

vostro sovrapiacente orrato affare

compiuto di ben tutto oltra pensare

di mortal cor magn'e mirabel cos $\underline{a}$,

sorprese... (c. 16, v. 1-4)

Soprapiacente donna, di tutto compiuto savere, di pregio coronata, degna mia Donna Compiuta, Guittone, vero devotissimo fedel vostro, de quanto el vale e pò, umilmente se medesmo racomanda a voi.

Gentil mia donna, l'onnipotente Dio mise in voi sì meravigliosamente compimento di tutto bene, che maggiormente sembrate angelica criatura che terrena, in ditto e in fatto e in la sembianza vostra tutta, ché, quanto omo_vede de_voi ${ }_{2}$ sembra_mirabil cosa_a_ciascuno bono conoscidore. Per che non degni fummo che tanta preziosa e mirabile figura, come voi siete, abitassc intra l'umana generazione d'esto seculo mortale; ma credo che piacesse a Lui di poner vo' tra noi per fare meravigliare... (I. V, $\$ 1-3$ )

4. Par exemple Stefano Carrai, La lirica toscana del Duecento, Roma-Bari, Laterza, 1997, p. 16 : «Alla Compiuta Donzella di Firenze [...] Guittone indirizzò con tutta probabilità la quinta delle sue epistole in prosa".

5. Guirtone d'Arezzo, Lettere, p. 86. 
de la lettre, "Frère Guittone ", c'est-à-dire le poète après son entrée dans l'ordre des Gaudenti? C'est peu probable, et ce pour deux raisons. D'une part on sait que la rubrique "Frère Guittone " est moins une indication sur l'état de clerc ou de laïc du poète au moment où celui-ci écrit, qu'une information sur le contenu moral et religieux des ouvres (les ouvres courtoises étant précédées de la simple rubrique «Guittone " $)^{6}$. D’autre part l'état de frère s'accorde assez mal avec la suite de la lettre $V$ : le destinateur "Guittone " se qualifie lui-même de fidèle tout dévoué à sa dame, et il loue celle-ci non seulement pour ses paroles et ses actes, mais aussi pour sa beauté physique (cf. $\$ 1$ et 2 note 3 ). Nous proposerons donc l'hypothèse suivante. Ce "Guitton " pourrait être le même que celui apparaissant dans le sonnet d'amour $\mathbf{n}^{\circ} 59$ où la dame s'adresse à son amant en l'appelant "Guitton " ${ }^{7}$, le même que celui placé en tête des sonnets de la tenson avec la "villana donna" (sonnets 80-86), et le même que celui se cachant vraisemblablement derrière l'initiale " $G$ » placée en tête des sonnets de la tenson $n^{\circ} 37-49$ : un personnage littéraire, un amant courtois de littérature qui, comme les amants locuteurs des deux chansons, met en avant sa fidélité topique. Un personnage fictif s'adressant à une dame fictive dans une lettre qui, pour Margueron, est elle aussi fictive. Reste la suite du texte qui differe cependant du type du discours courtois : le fidèle Guittone, en effet, invite sa dame non pas à l'aimer lui-même, mais à servir et à aimer Dieu. Ce locuteur serait donc semblable à celui des chansons - un amant courtois - mais un amant déjà engagé sur le chemin de la conversion. La proposition de $\mathrm{Cl}$. Margueron, que nous évoquerons ultérieurement, de reconnaître dans la lettre un "salut d'amour » transposé en prose conformément aux canons les plus stricts de l'épistolographie va dans le même sens : elle suggère elle aussi de voir dans le locuteur de la lettre un amant courtois.

Au niveau stylistique on peut remarquer, sinon d'autres points communs, du moins un brouillage des caractéristiques propres à chaque genre qui vient s'ajouter à la tendance à rythmer la prose épistolaire évoquée dans

6. Cl. Margueron dit que la subdivision Guittone/frère Guittone est juste dans l'ensemble, mais qu'elle nécessite néanmoins certaines retouches. Ibid., Introduzione, p. XIV.

7. Certo, Guitton, de lo mal tuo mi pesa (v. 1), GuirTone d'Arezzo, Canzoniere, sonetti d'amore del codice laurenziano, a cura di Lino Leonardi, Torino, Einaudi, 1994. l'introduction. La lettre $V$ est pratiquement dépourvue de sentences et de vérités générales et n'abonde pas en exclamations et interrogations, alors que la fréquence des unes et des autres est un des caractères de la prose de Guittone ${ }^{8}$. En revanche les sentences et vérités générales constellent les deux chansons $(1$, v. $5-6 ; 28-29 ; 37-42 ; 67-70 ; 72-74 ; 82-84 ; 102-104$; $114-115$. 16 , v. $25-26 ; 34-36 ; 41-43 ; 58-60)$, et la chanson 1 contient plusieurs interrogations emphatiques (v. 7-9; 16-18;22-25;30;71). Dans cette dernière le poète cite même des autorités (Polyclète v. 12 et l'Auteur - sans doute Salluste - v. 72) tandis que l'épistolier n'en cite aucune dans la lettre $V^{9}$. Ces traits propres aux genres didactiques mettent nos deux chansons à mi-distance entre la poésie lyrique et la poésie morale.

$\infty$

La fonction essentielle de la chanson 1 est suggérée par les premiers ver de son envoi : c'est un hommage à Corrado da Sterleto, un des feudataires de Frédéric II et de Manfred, qui a la particularité d'avoir cherché à favoriser la diffusion en Italie de la poésie en langue d'oc, en commandant à Uc Faidit - connu par ailleurs comme Uc de Saint-Circ - une grammairc de provençal et un dictionnaire de rimes : le Donatz Proensals (vers 1239. $1245)^{10}$. Dans cette chanson Guittone va montrer au représentant de la cour sicilienne et amateur de poésie provençale qu'il est non seulement un fin connaisseur de Giacomo da Lentini ${ }^{11}$, mais aussi plus généralement - et c'est ce qui nous intéresse ici - un expert en poésie courtoise : grâct à la rime riche entre les vers 21 et 115 qui établit une forte identité entre

8. Cesare SEGRE, "La sintassi del periodo nei primi prosatori italiani (Guittone Brunetto, Dante) ", in Lingua, stile e società, Milano, Feltrinelli, (1963'), 1991 p. $108-109 ; 112 ; 151$ et suivantes.

9. Cl. Margueron cite également les vers 1-7 comme exemple d'emploi d'une idée générale dans l'exorde, emploi conseillé par les dictatores de Bologne. Les vers 114 115 forment une conclusio a proverbio, autre précepte rhétorique (in Recherches sur Guittone d'Arezzo, Paris, PUF, 1966, p. 369)

10. Sur Corrado da Sterleto, cf. Cl. Margueron, Recherches..., p. 178-179, et L. Leonardi (in Guittone D'Arezzo, Canzoniere, p. xv), qui confirme à cet endroi l'identification entre Uc Faidit et Uc de Saint-Circ.

11. Cf. L. Leonard, "Tradizione e ironia nel primo Guittone : il confronto con Siciliani ", in Guittone d'Arezzo nel settimo centenario..., en part. p. 138-148. 
le mauvais «fenidore» (tel que Guittone se définit lui-même pour expliquer à Corrado qu'il ne l'a pas loué directement de crainte de ne pas être capable de "finir ", au sens d'achever et de parfaire, son panégyrique) et le "bon conoscidore" de la dame courtoise, Guittone revendique clairement sa qualité d'expert. Il la prouve en évoquant dans chaque strophe une situation courtoise. En effet, contrairement à l'impression d'ensemble donnée par les deux strophes liminaires, la chanson est moins une louange de la dame s'achevant sur une requête amoureuse, qu'une série de morceaux de bravoure ${ }^{12}$

Dans la première strophe le poète-amant construit sa louange autour du topos de la beauté miraculeuse de la dame. Celle-ci est d'abord « la fleur de la beauté de ce monde" (v. 7-8), " cœur ne peut concevoir, ni langue exprimer ce qui en [elle] pourrait être plus beau " (v. 13-15), c'est «un être $[\ldots]$ extraordinaire, [...] qui dépasse la nature " (v. 16-18), enfin « ce qu'on connaît et voit [d'elle] paraît [...] miraculeux " (v. 19-21). En vertu des règles courtoises codifiées par André le Chapelain qui disent qu'amour naît de la vue de la beauté - règle rappelée ici aux vers 5-6: " car d'une vue plaisante on sait assurément que naît amour »-il n'y a "point de merveille " qu'amour ait pris le poète, « mais c'est un miracle presque qu'il n'ait pris le cœur de tout homme» (v. 2-3). À travers ce travail sur mirabilia et miraculum qui cite à la fois des Provençaux et des Siciliens ${ }^{13}$, et en faisant nettement référence au De amore, Guittone se montre effectivement bon connaisseur de la poésie et des codes courtois.

Dans la deuxième strophe l'amant change de sujet : il ne s'adresse plus à la dame, mais s'interroge sur la manière dont il doit réagir quand cette dernière récompense son amour. La question qu'il pose est digne des questions posées dans le De amore:

Quale donque esser deo

poi tale donna intende il meo preghero,

e merta volontero

a cento dobli sempre il meo servire? ${ }^{14}$ (v. 22-25)

12. Vincent Moleta, The Early Poetry of Guittone d'Arezzo, London, The modern humanities research association, 1976, p. 105.

13. L. Leonardi, Guirtone d'Arezzo, Canzoniere, p. xxII-xXIII.

14. Les textes étant traduits en annexe nous ne donnons pas ici la traduction des passages longs.
Il y répond lui-même en brodant sur le lieu commun « mourir de joie » et, au-delà, sur la maladie d'amour : l'amour non partagé menant à la mort et l'amour partagé conduisant à mourir de joie. La strophe est construite autour de deux champs sémantiques: mourir/guérir et joie/douleur ${ }^{15}$. C'est une variante de l'oxymore « je meurs »/« je vis » que l'on trouve par exemple dans la canso de Folquet de Marseille que traduit le Giacomo da Lentini cité ici ( $A$ vos, midons, voill rettrair'en cantan).

Dans les trois strophes suivantes le poète-amant revient au rapport amoureux entre sa dame et lui-même. La troisième strophe est une variation sur le topos de la fidélité et de l'assujettissement absolus de l'amant à la dame :

Per che tutto me doe

voi, cui più che meo soe.

Meo non son già, ch'a far vosto piacere;

ché volonter isfareime in persona,

per far cosa di mene,

che piò stesse vo bene :

ché già non m’osa unqu'altro esser a voglia,

ch'ubedir vostra voglia. (v. 51-58)

La quatrième tourne autour du couple serviziolmerto. Quoi que fasse l'amant son service d'amour est toujours indigne de l'honneur que la dame lui fait en le récompensant. La cinquième et dernière strophe énumère les insuffisances traditionnelles de la louange : louer la dame n'apaise pas mais conduit au contraire à parler d'elle encore plus; cela ne permet pas d'exprimer correctement son asservissement et son désir; enfin, cela ne permet pas d'atteindre le plaisir ${ }^{16}$.

15. Non morto son (v. 27); morte sofferire (v. 29); se stesso consumare (v. 38); aucidereno (v. 40).

Lo meo guerire (v. 30); soccorgo (v. 35); guerisce (v. 38) et gueriscon (v. 40) qui, en début de vers, s'opposent à "se stesso consumare" et "aucidereno " en fin de vers. Gioia, dolzore (v. 27), gioia (v. 30), dolze (v. 40).

Dolore (v. 28), dolor (v. 35), cose molto amare (v. 39).

16. Eo non posso apagare

a dir, donna, de voi l'animo meo;

ché, se m'aiuti Deo,

quanto più dico, più talento dire; 
Dans cette chanson Guittone ne se contente pas de rivaliser avec les poètes courtois. Il lui arrive non seulement de souligner certaines impasses auxquelles conduisent les codes de la courtoisie, mais aussi de proposer des solutions pour les résoudre. C'est le cas notamment dans la deuxième strophe: comme nous l'avons vu, lorsque la dame récompense le service amoureux, l'amant court le risque de mourir de joie, ce qui mettrait un terme définitif à la courtoisie. Comment faire pour éviter cette impasse? C'est ici le poète moral qui intervient en expliquant comment il tempère la joie avec la douleur. C'est un savant dosage de l'une et de l'autre qu'il justifie au moyen d'une sentence empruntée à la vie quotidienne :

Ché de troppa grassia

guerisce om per se stesso consumare,

e cose molto amare

gueriscon zo che dolze aucidereno

de troppo bene è freno

male, e de male troppo è benenanza. (v. 37-42)

L'impasse évoquée dans la quatrième strophe - où jamais le service amoureux ne compense suffisamment et honorablement le guerdon accordé par la dame - est résolue de façon étrange dans un contexte courtois. L'artitude bienveillante de la dame qui fait de son amant-vassal son pair lui servira à entrer dans le royaume de Dieu. En conduisant le grand à estimer et grandir le petit, la courtoisie, par nature étrangère aux préceptes chrétiens, est intégrée à la morale religieuse :

Ma bon conforto m'ene

che, con più alto tene

segnor suo servo, più li po valere;

ché non po l'om capere

e non po dimostrare

la lingua mea com'è vostro lo core:

per poco non ven fore

a direve lo so coral desire.

E a ciò che 'n servire

potesse devenire

en quale loco piò fosse maggiore... (v. 85-95) sol per servire en la magion de Deo,

sì como sento e veo;

ma bona fede e gran voglia en piò fare

l'aiuta e 'l fa poggiare,

ché voglia e fe tal Di' ha fatto valere. (v. 76-84)

Les diverses impasses auxquelles conduit la louange courtoise qui n’apporte ni l'apaisement de l'âme, ni l'apaisement du corps (strophe v) sont résolues cette fois dans une direction totalement opposée à celle que nous venons de voir, puisque Guitıne propose comme solution «che l'amistà nostra de fatto/ormai, donna, s'usasse» : que notre amitié, ma dame, se traduise désormais dans les fait: (v. 96-97). C'est sur cette proposition sans équivoque, malgré la litote, que s'achève la chanson 1.

La chanson 16 est une louange qui vise avant tout, comme l'indiquent ses cinq premiers vers, les qualités morales de la gentil donna. La fin de la strophe signale l'hypothèse que le poète a choisi d'envisager ici : le cas où la dame s'est souciée d'apaiser la folie amoureuse de son amant, c'est-à-dire le cas où elle a accepté l'amitić « de fait " proposée à la fin de la chanson 1 pour sortir des impasses où conduisair la louange courtoise. La louange s'accompagne d'un bilan de cette hypothèse. En effet, en même temps que l'amant loue les qualités courtoises que sa dame a manifestées en lui accordant la récompense désirée, il révèle l'échec courtois de cette solution. C'est ce que nous allons détailler maintenant.

Les qualités morales louées par l'amant sont des qualités courtoises traditionnelles : ce sont la grande courtoisie de son cour doux et débonnaire (v. 8-9), la perfection du don qu'elle lui a accordé (v. 17), l'entière pitié qu'elle a manifestée à son égard (v. 24). La manière dont il les loue relève aussi de la poésie courtoise : pour mieux faire valoir les qualités de sa dame il leur oppose systématiquement sa propre indignité.

E non già meo valore

me conquistò de voi la segnoria,

ma la gran cortesia

de vostro dolze e debonaire core. (v. 6-9)

Gentil mia donna, amor, s'i per un cento avesse magno cor, forz'o savere, 
operandol sol sempre in voi valere, prendendovi final consomamento,

vostro compiuto don non mertaria. (v. 13-17)

Gentil mia donna, dono è ch'al fattore

è magno e a chi 'l prende è quasi nente;

ma 'I vostro dono, amor, non fo parvente,

ch'eo presi vita e voi donaste onore. (v. 25-28)

Comme l'indique ce dernier vers, cependant, le comportement courtois de la dame a causé la perte de ses qualités courtoises. En se comportant honorablement elle s'est finalement déshonorée: sa noblesse s'est abaissée (v. 10-11), et son grand honneur s'est transformé en honte (v. 21). Son attitude est finalement qualifiée de faute ("fallanza ", v. 29). L'amant a beau louer la courtoisie de sa dame, sa louange, comme il le dit lui-même, ne parvient pas à effacer cette faute. S'il se trouve en outre que ce comportement bienveillant vienne à l'oreille de « malparlieri », l'honneur de la dame est définitivement perdu (v. 37-43). Lamant a beau souhaiter mourir il ne peut rien changer à ce qui est advenu. Il le dit en tête de la dernière strophe et le répète en tête de l'envoi : Gentil mia donna, amor, non mi val voglia (v. 49), Gentil mia donna, non possa valere (v. 61). Le résultat de cette hypothèse courtoise est donc entièrement négatif.

Dans la lettre $V$ Guittone envisage une hypothèse diamétralement opposée à celle tentée dans la chanson 16. Reconnaître le service amoureux d'un amant-vassal et le récompenser en le « servant » à son tour aboutissant, non pas à s'élever et à entrer au paradis comme le suggéraient les vers 74-84 de la chanson 1, mais à déchoir comme l'ont répété toutes les strophes de la chanson 16, l'épistolier propose à la dame courtoise un service consistant à servir et à aimer Dieu de tout l'amour de son coú et avec une fidélité pure et parfaite $(\$ 4)$. Le paradoxe de cette lettre est que cette hypothèse est présentée comme une requête faite à la dame par son "vero devotissimo fedele " $(\$ 1)$, c'est-à-dire son serviteur habituel - l'amant des chansons 1 et 16 entre autres - qui introduit cette requête après avoir longuement loué la dame en des termes identiques à ceux qu'il utilisait dans les chansons courtoises ( $\$ 2$ déjà cité). La donna compiuta est un miracle, une créature angélique envoyée sur terre pour émerveiller l'Homme et lui servir de modèle. Le serviteur ne la loue pas en ces termes pour obtenir quelque chose en propre, mais pour l'inciter à servir Dieu afin de lui rendre grâce pour les dons dont Il l'a comblée $(\$ 5)$. Ce service élève celui qui le fait, préserve et parfait son honneur. Il faut servir Dieu en effet :

Acciò che voi siate nella corte di Paradiso altressì meravigliosamente grande come siete qui tra noi, e perché l'onorato vostro cominciamento e mezzo per preziosa fine vegna a perfezione de compiuta laude. $(\$ 6)$

Dans le cas contraire la dame encourt le même dommage que celui auquel s'était exposée la dame de la chanson 16, comme le montrent les syntagmes similaires qui évoquent ce risque. Pour la donna compiuta :

[...] Troppo fora periglisoso dannaggio e perta da pianger sempre mai senza alcun conforto, se per defetto vostro falliste a perfetta e onorata fine. $(\$ 7)$

Pour la donna gentile :

Donqua men danno fora

de la morte d'un om, si com'io, basso,

Ch'un sol punto d'onor foste fallita. (v. 44-46)

Cette lettre palinodique, par rapport aux hypothèses envisagées dans les chansons 1 et 16, rivalise clle aussi avec un modèle provençal : le salut d'amour. C'est au spécialiste de Guittone qu'est Claude Margueron que l'on doit d'avoir reconnu dans celle-ci un salut d'amour transposé en prose conformément aux canons les plus stricts de l'épistolographie ${ }^{17}$. À première vue en effet ce modèle sous-jacent n'est pas très évident, car il n'est pas facile de reconnaître dans la lettre $\mathrm{V}$ les caractéristiques du genre provençal. Celui-ci consiste en une variété d'épître en vers s'articulant en trois parties : une introduction comportant une salutation; l'épître amoureuse proprement dite composée pour sa part d'une longue description physique et morale louant la dame et d'une prière courtoise alternant avec la description ou se mêlant à elle; une conclusion contenant le plus souvent un salut final. La prière courtoise vise à amener la dame à manifester moins

17. Cf. Recherches sur Guittone..., p. 177-178, et son édition des Lettere, p. 86-87. 
de rigueur et à daigner aimer celui qui l'en prie ${ }^{18}$. Si l'on identifie sans peine dans la lettre V l'introduction $(\$ 1)$ et la description laudative des qualités physiques et morales de la dame $(\$ 2$ à 4$)$, il n’y a aucune prière courtoise visant à obtenir l'amour de celle-ci ${ }^{19}$. Comme nous l'avons souligné précédemment, le fidèle serviteur prie la dame non pas de l'aimer lui-même, mais d'aimer Dieu. Cet enseignement moral différent du salut d'amour et de la lettre V explique peut-être pourquoi Guittone a effectué une transposition. S'il s'était agi d'un discours courtois traditionnel (une louange s'achevant en requête amoureuse), le poète aurait pu transformer sans peine une chanson en salut, mais comme le discours courtois vise ici à convertir, la lettre comme genre didactique était sans doute plus adaptée.

Quoi qu'il en soit, cette transposition requiert de Guittone une bonne connaissance du salut d'amour. Aussi n'est-il pas inutile de préciser, pour conclure, que Uc de Saint-Circ, à qui s'était adressé Corrado da Sterleto, et qui a été cité comme point de référence pour le formalisme de Guittone ${ }^{20}$, parce qu'il visait comme notre poète "à démasquer l'idéologie du service d'amour, en la réduisant à sa réalité effective de jeu littéraire $~^{21}$, est vraisemblablement aussi l'auteur d'un salut d'amour ${ }^{22}$.

18. Pierre BeC, Les saluts d'amour du troubadour Arnaud de Maretil, Toulouse, Privat, 1961.

19. Pour Cl. Margueron la rigueur que l'amant tente habituellement d'adoucir « se métamorphose chez l'épistolier arétin en un péché capital, la femme consciente et fièrc de son excellence risquant de se rebeller contre Dieu, si elle ne sait reconnâtre sa dépendance à l'égard du dispensateur de tous les bienfaits » (Recherches..., p. 177).

20. Adolfo GASparY, La scuola poetica siciliana nel secolo XIII, Livorno, 1882, p. 163. Indication de Lino Leonardi dans son édition du Canzoniere de Guittone, p. xviri.

21. Maria Luisa Meneghetri, $l l$ pubblico dei trovatori. Ricezione e riuso dei testi lirici cortesi fino al XIV secolo, (1984), Torino, Einaudi, 1992, p. 140.

22. Bella donna gaja e valentz in A. Jean roy, Poésies de Uc de Saint-Circ, Toulouse, Privat, 1913 , p. 143 et suivantes.
Bien que les chansons 1 et 16 et la lettre $V$ appartiennent à des genres littéraires différents, et bien que leur sujet ne soit pas identique, les trois textes sont unis par des liens beaucoup plus profonds que les syntagmes communs soulignés par E. Pasquini. Les deux chansons et la lettre ont en commun d'abord d'être des exercices de style rivalisant avec des formes de la poésie provençale : la chanson courtoise et le salut d'amour. Ce sont des exercices d'émulation à travers lesquels Guittone prouve aussi sa connaissance et sa maitrise des codes courtois. Les deux chansons et la lettre partagent d'autre part une situation d'énonciation similaire : un amant courtois s'adresse à une noble dame qu'il commence par louer en des termes semblables dans les trois textcs, avant de lui adresser une prière. Les prières varient, proposant une série d'hypothèses de comportement courtois qui sont évaluées dans un même texte ou d'un texte à l'autre et qui aboutissent à une condamnation implicite de la courtoisie. L'amour et le service qui élèvent et qui honorent, ceux dont une dame peut attendre une juste récompense, consistent à aimer et servir Dieu.

Les trois textes se distinguent donc essentiellement par l'issue morale proposće à la dame. 


\section{Annexe}

\section{Annexe}

\section{Guittone d'Arezzo, Canzone I}

\section{Guitrone d'Arezzo, Chanson 1}

Se de voi, donna gente,

m'ha preso amor, no è già meraviglia, ma miracol somiglia

come a ciascun no ha l'anima presa; ché de cosa piacente

savemo de vertà ch'è nato amore.

Or da voi, che del fiore

del piacer d'esto mondo sete appresa, com po l'om far defesa?

fo di formare voi, co 'l bon pintore

Policreto fo de la sua pentura;

che non po cor pensare,

né lingua devisare,

che cosa in voi potesse esser piò bella.

Ahi, Deo, co sì novella

pote a esto mondo dimorar figura,

chè de sovra natura?

Ché ciò che l'om de voi conosce e vede

semiglia, per mia fede,

mirabel cosa a bon conoscidore.

Quale donque esser deo, poi tale donna intende il meo preghero, e merta volontero

a cento dobli sempre el meo servire?

Cert'ho miracol, ch'eo

non morto son de gioia e de dolzore;

ché, como per dolore,

\section{Si de vous, noble dame,}

amour m'a pris, point de merveille, mais c'est un miracle presque qu'il n'ait pris le coeur de tout homme; car d'une vue plaisante

on sait assurément que naît amour;

comment donc de vous,

qu'on considère comme la flcur de la beauté de ce monde, est-il possible de se défendre?

Car la nature a entendu

vous créer comme le bon peintre

Polyclète a créé sa peinture,

ainsi cour ne peut concevoir,

ni langue exprimer

ce qui en vous pourrait être plus beau.

Dieu! Comment un être

si extraordinaire, qui dépasse la nature,

peut-il avoir ici-bas sa demeure?

Car ce qu'on connaît et voit de vous

paraît, ma foi,

miraculeux à ceux qui sont bons juges.

Comment dois-je donc être,

dès lors qu'une telle dame entend ma prière,

et récompense volontiers

aux cent doubles toujours mon service?

C'est miracle vraiment

que je ne sois mort de joie et de douceur,

car, comme de douleur, 
po l'om per gioia morte sofferire.

Ma che? Lo meo guerire

è stato con schermire,

ver zo mettendo tutta mia possanza;

ché quando troppo la sento abondare,

mantenente m'acorgo

e con dolor socorgo,

quale me credo che maggiore sia.

Ché de troppa grassia

guerisce om per se stesso consumare,

e cose molto amare

gueriscon zo che dolze aucidereno:

de troppo bene è freno

male, e de male troppo è benenanza.

Tantosto, donna mia,

com eo vo vidi, foi d'amor sorpriso,

né già mai lo meo viso

altra cosa che voi non devisoe.

E sì m'è bon ch'eo sia

fedele voi, che 'n me non trovo cosa

ver ciò contrariosa,

che l'alma e lo saver deletta cioe.

Per che tutto me doe

voi, cui più che meo soe.

Meo non son già, ch'a far vostro piacere;

ché volonter isfareime in persona,

per far cosa di mene,

che piò stesse vo bene:

- ché già non m'osa unqu'altro esser a voglia,

ch'ubedir vostra voglia;

e s'eo de voi disio cosa alcona,

sento che savi bona

e che valor v'accresce in allegranza.

De tale disianza

non piaccia a Deo ch'io mai possa movere. on peut mourir de joie.

Mais comment ai-je fait? J'ai guéri

en me défendant,

utilisant à cela toutes mes forces :

quand je sens la joie déborder,

je m'en aperçois aussitôt

et j'y remédie avec une douleur

que je crois plus forte.

On guérit de trop de graisse

en se consumant soi-même,

ainsi une grande amertume

guérit-elle ce que tuerait la douceur :

le mal est un frein à l'excès de bien,

et le bien à l'excès de mal

Aussitôt que je vous vis,

ma dame, je fus pris d'amour,

et jamais mon regard

ne regarda un autre objet que vous.

Être votre fidèle me plaît si fort

qu'en moi je ne trouve rien

qui s'y oppose,

car cela réjouit mon âme et mon esprit.

Aussi me donné-je tout à vous,

à qui j'appartiens plus qu'à moi-même.

Je ne suis moi-même qu'en faisant votre bon plaisir;

volontiers je déferais mon être

pour faire de moi un objet

qui vous agréerait mieux :

car je ne peux désormais avoir d'autre désir

que d'obéir à votre désir.

Et si je veux quelque chose de vous

je sens qu'elle vous est agréable

et accroît votre valeur dans la joie.

De ce désir, à Dieu ne plaise

que jamais je ne puisse m'éloigner. 
Per tutto ciò non servo,

né porea mai servir, l'onor né 'l bene, che per voi fatto m'ène;

ché troppo è segno d'amoroso amore

far lo signor del servo

su' par; ed è ben cosa che non mai

pot'om mertare assai.

Donque como de merto avrò onore?

Ché sì como l'Autore

pon, ch'amistà di core

è voler de concordia e desvolere,

faite voi me, ché zo volete ch'eo.

Ma bon conforto m'ène

che, con più alto tene

segnor suo servo, più li po valere;

ché non po l'om capere

sol per servire en la magion de Deo,

sì como sento e veo

ma bona fede e gran voglia en piò fare l'aiuta e 'l fa poggiare,

ché voglia e fe tal Di' ha fatto valere.

Eo non posso apagare

a dir, donna, de voi l'animo meo;

ché, se m’aiuti Deo,

quanto più dico, più talento dire;

e non po dimostrare

la lingua mea com'è vostro lo core:

per poco non ven fore

a direve lo so coral desire.

Ed a ciò che 'n servire

potesse devenire

en quale loco piò fosse maggiore,

vorrea che l'amistà nostra de fatto

ormai, donna, s'usasse;

ché, se per me s'osasse

dir, troppo tarda enver ciò dimorate.
Pour autant je ne sers

ni ne pourrais jamais servir à la mesure de l'honneur et du bien que vous me dispensez;

car, que le scigneur fasse de son serviteur un égal, est d'amoureux amour une trop grande marque;

et c'est là un bienfait que jamais

on ne peut rendre suffisamment.

Comment donc tirerai-je honneur de vous le rendre?

En effet, comme l'Auteur écrit que

l'amitié de cour c'est vouloir

et ne pas vouloir en harmonie,

ainsi agissez-vous avec moi, car votre volonté est la mienne.

Mais je suis réconforté de ce que,

plus un seigneur place haut son serviteur,

plus il peut en retirer de valeur,

car l'homme ne peut entrer dans la maison de Dieu,

à ce que j'entends et vois,

grâce à son seul service;

mais une foi bonne et un grand désir d'en faire plus

l'aident et le font s'élever.

car Dieu a donné de la valeur à ce désir et à cette foi.

Je ne peux apaiser

mon âme en parlant de vous, ma dame,

car, Dieu m’est témoin,

plus je parle de vous, plus je désire parler;

mais ma langue ne peut montrer

combien mon coeur est à vous :

peu s'en faut qu'il ne sorte

pour vous dire son désir ardent.

Et afin qu'en servant

je puisse parvenir

en plus haut lieu,

je voudrais que notre amitié

se traduise désormais, ma damé, dans les faits;

car, si j'osais le dire,

vous tardez à l'excès en cette affaire. 
Ché de fare amistate

certo lo tardare pareme mat to;

e comperato accatto

non sa sì bon, como quel ch'è 'n don priso;

e sì como m’è viso,

endugio a grande ben tolle savore.

Currado da Sterleto, mea canzone

vo mando e vo presento,

ché vostro pregio vento

m'ha voi fedele e om de ciò ch'eo vaglio;

e se non mi travaglio

de vostro regio dir, quest'è cagione,

che bene en sua ragione

non crederea già mai poter finare :

non dea l'om comenzare

la cosa, onde no è bon fenidore.
Tarder à devenir amis

me paraît chose folle;

et un bien acheté

n'agrée pas autant qu'un bien reçu en don

et il m'est avis

que le retard ôte toute saveur à un grand bienfair.

Corrado de Sterletto, cette chanson,

je vous l'envoie et vous la présente,

car votre mérite m'attache à vous

comme votre fidèle (serviteur) et votre homme, pour ce que je vaux et si je ne fais nul effort

pour chanter votre mérite, c’est qu'un éloge

en rapport avec votre valeur,

je ne croirais jamais pouvoir l'achever :

on ne doit pas commencer

une entreprise que l'on ne sait mener à bonne fin. 


\section{Gurttone D'Arezzo, Canzone 16}

Gentil mia donna, gioi sempre gioiosa, vostro sovrapiacente orrato affare compiuto di ben tutto, oltra pensare di mortal cor magn'e mirabel cosa, sorprese l'alma mia de tutto amore; e non già meo valore

me conquistò de voi la segnoria, ma la gran cortesia

de vostro dolze e debonaire core non già guardando ciò, ched in bassezza

tornasse vostr'altezza,

ma solo de quetar lo meo follore.

Gentil mia donna, amor, s'i per un cento avesse magno cor, forz'o savere,

operandol sol sempre in voi valere,

prendendovi final consomamento,

vostro compiuto don non mertarì;

ché la passione mia

fo de natura fellonesca tanto,

non mai partia de pianto

scnza tornar vostro onor grande a onta.

Voi lo tornaste, amore, e non v'adusse

cosa ch'a mala vista fusse,

ma solo l'alma d'ogne pietà ponta.

Gentil mia donna, dono è ch'al fattore è magno e a chi 'l prende è quasi nente; ma 'l vostro dono, amor, non fo parvente, ch'eo presi vita e voi donaste onore.

Ma certo, amore meo, d'esta fallanza

v'aduce perdonanza

lo bisogno o' fui, grande oltra ch'eo dico;

ché non ho già nemico

sì fero, ch'a pietà non fosse dato.

\section{Guittone d'Arezzo, Chanson 16}

Ma noble dame, joyau toujours joyeux,

votre noblesse honorée et par-dessus tout plaisante,

en tout point accomplie, plus grande et plus admirable

que ne peut l'imaginer un cocur de mortel,

a saisi mon âme de tout l'amour possible;

et ce n'est pas ma valeur personnelle

qui me permit d'obtenir que vous fussiez ma suzeraine,

mais la grande courtoisie

de votre coeur doux et débonnaire

qui ne s'est pas soucié

que s'abaissât votre haute nollesse

mais seulement d'apaiser ma folie.

Ma noble dame, mon amour, si j'avais cent fois plus de coeur, de force et de sagesse,

et si je ne les urilisais que pour valoir plus devant vous jusqu'à m'y consumer tout entier,

je ne mériterais pas votre don parfait,

car ma passion

a été de nature fort vile

et n'a pas cessé de se plaindre

jusqu'à cc que votre grand honneur soit transformé en honte.

C'est vous qui l'avez transformé, mon amour,

poussée non pas par ce qui pourrait paraître mal,

mais seulement par votre âme que poignait toute la pitié du monde.

Ma noble dame, il est un don

grand pour qui le fait et presque insignifiant pour qui le reçoit,

mais votre don, mon amour, n'est pas de cette nature,

car j'ai reçu la vie et vous avez donné votre honneur.

Mais assurément, mon amour; le besoin où j'étais,

beaucoup plus grand que je ne le dis,

tel qu'il n'est d'ennemi

si cruel qui n'aurait eu pitié de moi,

excuse votre faute. 
Ché non è vizio, ma virtù, pietate; ma vizio è crudeltate

e contra del pietoso esser spietato.

Gentil mia donna, assai porea mostrare ragion, che porterea 'n vostra defesa; ma tuttavia vorrea morte aver presa e ciò ch'è stato fosse anco ad istare.

Ché poi che corse tra villana gente alcun parlar nesciente,

nulla scusa ostar mai l'omo ne pora.

Donqua men danno fora

de la morte d'un om, sì com'io, basso,

ch'un sol punto d'onor foste fallita;

ch'onor val più che vita,

per che pria morto esser vorria, lasso!

Gentil mia donna, amor, non mi val voglia; ma, se valesse, già parrebbe tanto,

che nel mondo no ha loco né canto, no li portasse pietanza e doglia;

che già fu me, gentil mia donna, noia vostr'amorosa gioia,

ver ch'ora mi serea destruggimento

d'onne crudel tormento,

potendo vo tornare in vostro stato :

ché dirittura vol che no schifare

deggiom pena portare,

unde possa mendar ciò ch'ha peccato.

Gentil mia donna, non poss'a valere, menderaggio a podere;

che com'eo vi servii de folle amore,

mai sempre a vostro onore

vo serverò de quant' eo so valere,

remosso onne villano intendimento;

e per simil convento

piacciavi, amor, mia fedeltà tenere.
Car la pitié n'est pas péché, nais vertu;

mais c'est la cruauté qui est péché,

et se montrer sans pitié vers qui est pitoyable.

Ma noble dame, je pourrais alléguer

de nombreux arguments pour votre défense; cependant je préférerais être mort

et que ce qui est arrivé soit encore à venir, car une fois que quelque sotte rumeur

a couru parmi des personnes viles,

plus jamais on ne pourra opposer aucune excuse.

Et donc, qu'un homme humble tel que moi

meure serait moins grave

que si votre honneur s'abaissait d'un seul degré.

Car l'honneur vaut plus que la vie

aussi, voudrais-je, hélas, plutôt être mort.

Ma noble dame, mon amour, ma volonté est vaine; mais, si elle n'était pas vaine, elle aurait un tel pouvoir qu'il n'y aurait de lieu ni de recoin au monde qu'elle n'emplirait de pitié et de douleur.

Je souffris, ma noble dame, autrefois, pour gagner la joie de votre amour,

aujourd'hui au contraire

tous mes cruels tourments

seraient détruits

si je pouvais vous rendre à votre état :

la justice veut qu'on ne répugne pas à endurer la peine pouvant réparer ce en quoi on a péché.

Ma noble dame, ne le pouvant comme je le veux,

Je réparerai autant que je le peux;

de même que je vous ai servie d'un amour fou,

désormais pour votre honneur

je vous servirai autant que je suis capable,

abandonnant tout vil dessein.

par un tel serment,

plaise à vous, mon amour, me garder pour fidèle. 


\section{Guittone D'Arezzo, Lettera $V$}

\section{FRATE GUITTONE}

1 Soprapiacente donna, di tutto compiuto savere, di pregio coronata, degna mia Donna Compiuta, Guitton, vero devotissimo fedel vostro, da quanto el vale e pò, umilemente se medesmo racomanda voi.

2 Gentil mia Donna, l'onnipotente Dio mise in voi sì meravigliosamente compimento di tutto bene, che maggiormente sembrate angelica criatura che terrena, in ditto e in fatto, e in la sembianza vostra tutta, ché, quanto omo vede de voi, sembra mirabil cosa a ciascuno bono conoscidore. 3 Per che non degni fummo che tanta preziosa e mirabile figura, come voi siete, abitasse intra l'umana generazione d'esto seculo mortale; ma credo che piacesse a Lui di poner vo' tra noi per fare meravigliare, e perché fuste ispecchio e miradore, ove se provedesse e agenzasse ciascuna valente e piacente donna e prode omo, schifando vizio e seguendo vertù; e per che voi siete deletto e desiderio e pascimento de tutta gente che vo vede e ode. 4 Or dunque, gentile mia Donna, quanto el Signor nostro v'ha maggiormente allumata e smirata a compimento de tutta preziosa vertute più chaltra donna terrena, e cusì più ch'altra donna terrena devete intendere a Lui servire e amare de tutto corale amore e de pura e de compiuta fede. 5 E però umiliatevi a Lui, reconoscendo ciò ch'avete da Lui, in tal guisa che l'altezza dell'animo vostro, né la grandezza del core, né la beltà, né 1 piacere de l'onorata persona vostra non vo faccia obriare né mettere a non calere Lui che tutto ciò v'ha dato; 6 ma ve ne caglia tanto che 'l core e 'l corpo e 'l penseri vostro tutto sia consolato in Lui servire, acciò che voi siate indela corte di Paradiso altressì meravigliosamente grande come siete qui tra noi, e perché l'onorato vostro cominciamento e mezzo per preziosa fine vegna a perfezione de compiuta laude. 7 Ché troppo fora periglioso dannaggio e perta da pianger sempre mai senza alcun conforto, se per defetto vostro falliste a perfetta e onorata fine.

\section{GuitTone d'Arezzo, Letire $V$}

FRÈre GUITTONE

1 Dame par-dessus tout plaisante, au savoir en tout accompli, couronnée de valeur, ma digne Dane Accomplie, Guittone, votre sincère fidèle très fervent, autant qu'il en est capable ct le peut, humblement se recommande à vous.

2 Ma noble dame, le Dicu tout puissant mit si merveilleusement en vous tout bien accompli que vous paraissez une créature plus angélique que terrestre, dans vos paroles et dans vos actes, et dans toute votre personne, car tout ce qu'on voir de vous paraît miraculeux à ceux qui sont bons juges. 3 Aussi n'érions-nous pas dignes qu'un être aussi précieux et admirable que vous habite au milieu du genre humain de ce siècle mortel, mais je crois qu'il Lui a plu de vous mettre parmi nous pour nous émerveiller et pour que vous soyez le miroir et le modèle où puissent se contempler et se complaire toute dame valeureuse et belle et tout homme vaillant, méprisant ainsi le vice et suivant la vertu. Aussi êtes-vous le plaisir, le désir et la nourriture de tous ceux qui vous voient et vous entendent. $4 \mathrm{Or}$ donc, ma noble Dame, de même que notre Seigneur vous a plus parfaitement illuminée et éclairée do toutes les vertus précieuses que toute autre dame sur terre, de même plus que toute autre dame sur terre vous devez viser à le servir et à l'aimer de tout l'amour de votre coeur et avec une fidélité pure et parfaite. 5 Aussi inclinez-vous devant Lui en reconnaissant ce qui vous vient de Lui, de façon que ni la grandeur de votre âme, ni la largesse de votre coeur, ni la beauté, ni l'attrait de votre honorable personne ne vous fassent ni oublier ni négliger Celui qui vous a donné tout cela. 6 Qu'il vous importe, au contraire, au point que tout votre cour, votre corps et vos pensers soient heureux de Le servir, pour que vous soyez à la cour du Paradis aussi merveilleusement élevée que vous l'êtes ici-bas parmi nous, et pour que ce que vous avez honorablement commencé et continué s'accomplisse en une fin précieuse au milieu de louanges parfaites. 7 Ce serait un dommage trop grave et une perte à jamais regrettable sans nul soulagement, si par votre faute vous échouiez à atteindre une fin parfaite et honorable. 